\title{
APPROXIMATION PROPERTIES FOR DISCRETE GROUPS
}

\author{
JACEK BRODZKI AND GRAHAM A. NIBLO
}

\section{INTRODUCTION}

The reduced $C^{*}$-algebra $C_{r}^{*}(\Gamma)$ of a group $\Gamma$ (which we shall assume to be discrete) arises from the study of the left regular representation $\lambda$ of the group ring $\mathbb{C} \Gamma$ on the Hilbert space of square-summable functions on the group. Various important properties of the group can be expressed in terms of analytic properties of this algebra. We give a survey of the main points below but let us just mention the result of Lance [15] that the reduced $C^{*}$-algebra is nuclear if and only if the group is amenable.

From the point of view of noncommutative geometry, a $C^{*}$-algebra is always considered as an algebra of continuous functions on some space. In the case of the reduced $C^{*}$-algebra that space is a space of representations of the group. Unfortunately it is not easy to understand the structure of this algebra in general, though partial information is obtained by studying topological invariants of this algebra, for instance its $K$-theory. This, too, is complicated although the Baum-Connes conjecture postulates a possible way to compute it.

In some cases one can find an interesting smooth subalgebra of $C_{r}^{*}(\Gamma)$, that would play a role similar to the algebra of smooth functions on a manifold. This algebra of Schwartz-type functions is most useful when it has the same $K$-theory as $C_{r}^{*}(\Gamma)$, while being more accessible to homological methods. Algebras of this kind are normally defined by imposing a suitable growth condition on the space of functions. A very interesting example of when this can be done is provided by the class that satisfy the rapid decay property (RD), introduced by Jolissaint [11]. Here the control on growth is derived from a length function which turns the group $\Gamma$ into a metric space with interesting geometry.

Definition 1.1. A length function on a discrete group $\Gamma$ is a function $\ell: \Gamma \rightarrow \mathbb{R}$ taking values in the non-negative reals which satisfies the following conditions:

(1) $\ell(1)=0$, where 1 is the identity element of the group;

(2) For every $g \in \Gamma \ell(g)=\ell\left(g^{-1}\right)$.

(3) For every $g, h \in \Gamma, \ell(g h) \leq \ell(g)+\ell(h)$ for all $g, h \in \Gamma$,

A group equipped with a length function becomes a metric space with the left-invariant metric $d(g, h)=\ell\left(h^{-1} g\right)$. 
For any length function $\ell$ and a positive real number $s$ we define a Sobolev norm on the group ring $\mathbb{C} \Gamma$

$$
\|f\|_{\ell, s}=\sqrt{\sum_{\gamma \in \Gamma}|f(\gamma)|^{2}(1+\ell(\gamma))^{2 s}}
$$

Following Jolissaint [11] (see also [2]) we say that $\Gamma$ has the rapid decay property (property $R D$ ) with respect to the length function $\ell$ if and only if it satisfies the following property: There exist a $C>0$ and $s>0$ such that for all $f \in \mathbb{C} \Gamma$

$$
\|\lambda(f)\| \leq C\|f\|_{\ell, s}
$$

where the norm on the left hand side is the operator norm in $\mathfrak{L}\left(\ell^{2}(\Gamma)\right)$. This inequality indicates how the operator norm, which is in general difficult to compute, can be controlled by a more computable Sobolev norm. Examples of RD groups include hyperbolic groups [9], groups acting on CAT(0)-cube complexes [2] and co-compact lattices in $S L_{3}(\mathbb{R})$ or $S L_{3}(\mathbb{C})[14]$.

The purpose of this note is to provide an illustration of an interesting and nontrivial interaction between analytic and geometric properties of a group. We provide a short survey of approximation properties of operator algebras associated with discrete groups. We then demonstrate directly that groups that satisfy the property $\mathrm{RD}$ with respect to a conditionally negative length function have the metric approximation property, which is defined below. We obtain this result by combining two important ingredients. The RD property provides us with estimates for operator norms, while the properties of conditionally negative kernels allow us to define multipliers. These two properties together combine to control norm inequalities in the reduced $C^{*}$-algebra. Although this result is implied by a result of Jolissaint and Valette [12] we feel that this direct approach illustrates the important role played by the RD property rather well. For another short introduction to the Rapid Decay property and its interaction with multipliers (which play a key role in this note) we would like to draw the reader's attention to a short article by Indira Chatterji which appears as an appendix to $[17]$.

This note represents an extended version of the talk delivered by the first author at the meeting on ' $C^{*}$-algebras and elliptic theory', which took place in Będlewo in February 2004. J. Brodzki would like to thank the organizers for providing a very stimulating environment for exchanging ideas.

\section{Algebras associated With groups}

It is well known that all topological information about a compact Hausdorff space $X$ can be recovered from the unital abelian $C^{*}$-algebra $C(X)$ of continuous functions on $X$. Moreover, it is known that any 
commutative $C^{*}$-algebra is isomorphic to an algebra of continuous functions on a locally compact space $X$. This point of view has been developed with great success within noncommutative geometry, which provides the geometric, analytic and homological tools for the study of 'quantum spaces'. In this approach, $C^{*}$-algebras and their topological invariants are studied using methods modeled on classical topology and geometry.

When the space $X$ is equipped with some algebraic structure, for instance when $X$ is a locally compact group, one would hope to have a way of encoding, in operator-algebraic terms, both the topology and algebra of $X$. We shall outline briefly how this might be done.

Let us assume that $\Gamma$ is a discrete group. The group ring $\mathbb{C} \Gamma$ consists of all finitely supported complex-valued functions on $\Gamma$, that is of all finite combinations $f=\sum_{\gamma \in \Gamma} f_{\gamma} \delta_{\gamma}$ with complex coefficients $f_{\gamma}$ where $\delta_{\gamma}$ is the characteristic function of the set $\{\gamma\}$.

If we equip the group ring with the pointwise product of functions then the resulting *-algebra contains information, such as there is, about the topology of $\Gamma$ but completely ignores its group structure. To encode that information we need to use the convolution product defined for any $\gamma, \eta \in \Gamma$ by

$$
\delta_{\gamma} * \delta_{\eta}=\delta_{\gamma \eta}
$$

The left-regular representation $\lambda$ of the group ring $\mathbb{C} \Gamma$ assigns to each element $f \in \mathbb{C} \Gamma$ a bounded operator $\lambda(f)$ which acts on any $\xi \in \ell^{2}(\Gamma)$ by convolution:

$$
\lambda(f)(\xi)=f * \xi .
$$

The image $\lambda(\mathbb{C} \Gamma)$ of the group ring under the left-regular representation is a $*$-subalgebra of the algebra $\mathfrak{L}\left(\ell^{2}(\Gamma)\right)$ of bounded operators on $\ell^{2}(\Gamma)$.

Definition 2.1. The closure of $\lambda(\mathbb{C} \Gamma)$ in the $C^{*}$-norm topology of $\mathfrak{L}\left(\ell^{2}(\Gamma)\right)$ is by definition the reduced $C^{*}$-algebra of $\Gamma$ denoted $C_{r}^{*}(\Gamma)$.

The reduced $C^{*}$-algebra of the group $\Gamma$ does not arise from the topological structure of the group $\Gamma$ but rather contains information about the representation theory of $\Gamma$. The case of abelian groups illustrates this point rather well. For an abelian group $\Gamma$ the Pontryagin dual $\widehat{\Gamma}$ is by definition the group of characters, that is group homomorphisms from $\Gamma$ with values in the circle group $\mathbb{T}$. Then one has

$$
C_{r}^{*}(\Gamma)=C_{0}(\widehat{\Gamma}) .
$$

For example, when $\Gamma=\mathbb{Z}^{k}$, the dual group $\widehat{\mathbb{Z}^{k}}$ is the $k$-dimensional torus $\left(S^{1}\right)^{k}$. More generally, the reduced $C^{*}$-algebra corresponds to an algebra of continuous functions on the tempered dual of $\Gamma[21,22][1]$.

The reduced $C^{*}$-algebra of a group collects the information about the irreducible unitary representations that make up the left regular 
representation of the group ring. Dually, the data concerning the representation theory of a group is encoded in its Fourier algebra [4], [8] which we will now describe.

We recall first that a complex-valued function $\phi$ on $\Gamma$ is a coefficient function of the left regular representation iff

$$
\phi(\gamma)=\left\langle\lambda\left(\delta_{\gamma}\right) \xi, \eta\right\rangle
$$

for all $\gamma \in \Gamma$ and some vectors $\xi, \eta \in \ell^{2}(\Gamma)$.

Definition 2.2. The Fourier algebra $A(\Gamma)$ is the completion of $\mathbb{C} \Gamma$ in the norm

$$
\|u\|_{A(\Gamma)}=\inf \left\{\|\xi\|\|\eta\| \mid u(\gamma)=\left\langle\lambda\left(\delta_{\gamma}\right) \xi, \eta\right\rangle\right\} .
$$

With this norm, $A(\Gamma)$ is a Banach algebra with the pointwise multiplication.

\section{A BRIEF SURVEY OF APPROXIMATION PROPERTIES}

The study of approximation properties was initiated by Grothendieck in relation to the notion of nuclearity that he introduced in [6]. His fundamental ideas have been applied to the study of groups; in this case one discovers that important properties of groups, like amenability or exactness, can be expressed in terms of approximation properties of the associated operator algebras introduced in the previous section. We give here a brief overview of the main facts. Our main references are Wassermann's lecture notes [24] and Paulsen's text [20].

Let $A$ and $B$ be two $C^{*}$-algebras and $\phi: A \rightarrow B$ be a linear map. Then

$$
\phi \otimes \operatorname{id}_{M_{n}}: M_{n}(A) \rightarrow M_{n}(B), \quad\left(a_{i j}\right) \mapsto\left(\phi\left(a_{i j}\right)\right)
$$

is a linear map, denoted by $\phi_{n}$. If $\phi$ is a $*$-homomorphism then $\phi_{n}$ is also a $*$-homomorphism.

The map $\phi$ is said to be completely bounded iff $\phi_{n}$ is bounded for all $n \geq 1$. Let

$$
\|\phi\|_{c b}=\sup \left\{\left\|\phi_{n}\right\| \mid n \in \mathbb{N}\right\}
$$

When this expression is finite, it is called the completely bounded norm of $\phi$.

Similarly, the map $\phi$ is completely positive iff $\phi_{n}$ is positive for all $n$; it is completely isometric iff $\phi_{n}$ is isometric for all $n \geq 1$ and $\phi$ is completely contractive iff the maps $\phi_{n}$ are contractions $\left(\left\|\phi_{n}\right\| \leq 1\right)$ for all $n$.

Completely positive maps are completely bounded [24, 1.5]. When $A$ is unital and $\phi$ is a complete contraction, then $\phi$ is completely positive if and only if $\|\phi(1)\|=\|\phi\|$.

With these notions at hand we can introduce one of the main approximation properties. 
Definition 3.1. A $C^{*}$-algebra $A$ is nuclear iff it has the following completely positive approximation property (CPAP): The identity map id : $A \rightarrow A$ can be approximated in the point-norm topology by finite rank completely positive contractions. This means that that there exist nets of operators $T_{\alpha}: A \rightarrow M_{n_{\alpha}}(\mathbb{C})$ and $S_{\alpha}: M_{n_{\alpha}}(\mathbb{C}) \rightarrow A$ such that for all $a \in A$

$$
\lim _{\alpha}\left\|S_{\alpha} T_{\alpha}(a)-a\right\|=0
$$

Nuclear algebras satisfy in particular the metric approximation property (MAP) of Grothendieck which is stated as follows.

Definition 3.2. A $C^{*}$-algebra $A$ has the metric approximation property of Grothendieck iff the identity map on $A$ can be approximated in the point-norm topology by a net of finite rank contractions.

It is clear that CPAP implies MAP.

One of the most important examples of how approximation properties of algebras relate to properties of groups is provided by the following theorem of Lance [15].

Theorem 3.3. A discrete group $\Gamma$ is amenable if and only if its reduced $C^{*}$-algebra $C_{r}^{*}(\Gamma)$ is nuclear.

So we see that our group $\Gamma$ is amenable if and only if its reduced $C^{*}$ algebra has the CPAP. An alternative way to characterise amenability is via an approximation property for the Fourier algebra $A(\Gamma)$. Leptin proved in [16] that a locally compact group $G$ is amenable if and only if the Fourier algebra $A(G)$ has an approximate identity which is bounded in the norm $\|-\| \|_{A(G)}$.

In the case of free groups, Haagerup showed that the Fourier algebra $A\left(\mathbb{F}_{n}\right)$ has an approximate unit that is unbounded in the norm of the Fourier algebra, but is bounded in the so called multiplier norm.

Definition 3.4. A complex-valued function $u$ on $\Gamma$ is a multiplier for $A(\Gamma)$ if the linear map $m_{u}(v)=u v$ maps $A(\Gamma)$ into $A(\Gamma)$. The set of multipliers of $A(\Gamma)$ is denoted $M A(\Gamma)$. If $u \in M A(\Gamma)$ then $u$ is a bounded continuous function and $m_{u}$ is a bounded operator on the space $A(\Gamma)$.

We say that $u$ is a completely bounded multiplier if and only if the operator $m_{u}$ is completely bounded. The set $M_{0}(A(\Gamma))$ of completely bounded multipliers is equipped with the norm

$$
\|u\|_{M_{0} A(\Gamma)}=\left\|m_{u}\right\|_{c b}
$$

By analogy with Leptin's result we have the following definition of weak amenability.

Definition 3.5. We say that a group $\Gamma$ is weakly amenable iff $A(\Gamma)$ has an approximate identity that is bounded in the multiplier norm. 
Hence a group is weakly amenable if there is a net $\left\{u_{\alpha}\right\}$ in $A(\Gamma)$ and a constant $C$ such that $\left\|u_{\alpha} v-v\right\| \rightarrow 0$ for all $v \in A(\Gamma)$ and such that $\left\|u_{\alpha}\right\|_{M_{0} A(\Gamma)} \leq C$ for all $\alpha$.

We have thus defined a weak form of amenability via the Fourier algebra. Closing the circle it turns out that this property can also be formulated in terms of the completely bounded approximation property for the reduced $C^{*}$-algebra.

Definition 3.6. A $C^{*}$-algebra $A$ is said to have the completely bounded approximation property (CBAP) if there is a positive number $C$ such that the identity map on $A$ can be approximated in the point-norm topology by a net $\left\{T_{\alpha}\right\}$ of finite rank completely bounded maps whose completely bounded norms are bounded by $C$.

We have the following important result of Haagerup (see [8, p.669]).

Theorem 3.7. Let $\Gamma$ be a discrete group. Then the following are equivalent:

(1) $\Gamma$ is weakly amenable.

(2) $C_{r}^{*}(\Gamma)$ has the $C B A P$.

We have seen that a discrete group $\Gamma$ is amenable if and only if $C_{r}^{*}(\Gamma)$ is nuclear. It is natural to ask if there is a property of groups that corresponds to the CBAP, and the answer is provided by the notion of exactness, introduced by Kirchberg and Wassermann in [13].

Definition 3.8. We say that a discrete group $\Gamma$ is exact iff $C_{r}^{*}(\Gamma)$ is exact as a $C^{*}$-algebra: this means that the operation of taking the minimal tensor product with this algebra is an exact functor in the category of $C^{*}$-algebras.

Exact groups are known to admit uniform embeddings in a Hilbert space and therefore to satisfy the Novikov conjecture by an important result of $\mathrm{Yu}[26]$. Here we have a concrete application of noncommutative geometry to a classical problem in topology. It is important in our context because of the following theorem, due to Kirchberg and Wassermann.

Theorem 3.9. If a $C^{*}$-algebra $A$ satisfies the $C B A P$ then $A$ is exact.

We provide a proof that was kindly communicated to us by Ozawa.

Proof. Let $A$ be a $C^{*}$-algebra with the CBAP; this means that there exists a uniformly bounded family of completely bounded finite rank operators $T_{n}: A \rightarrow A$ such that for any $a \in A,\left\|T_{n}(a)-a\right\| \rightarrow 0$.

We need to show that for any exact sequence

$$
0 \rightarrow I \stackrel{i}{\rightarrow} B \stackrel{q}{\rightarrow} Q \rightarrow 0
$$

of $C^{*}$-algebras, the sequence

$$
0 \rightarrow A \otimes I \rightarrow A \otimes B \stackrel{\mathrm{id}_{A} \otimes q}{\longrightarrow} A \otimes Q \rightarrow 0
$$


is also exact, where $\otimes$ stands for the minimal tensor product.

We note first that for any $x \in A \otimes B$ we have $\left\|\left(T_{n} \otimes \mathrm{id}_{B}\right)(x)-x\right\| \rightarrow 0$. (Since the maps $T_{n} \otimes \mathrm{id}_{B}$ are uniformly bounded in $n$ it is enough to check this assertion on simple tensors $x=a \otimes b$.). Assume now that $x$ is an element of the kernel $\operatorname{ker}\left(\operatorname{id}_{A} \otimes q\right)$ of the quotient map $\mathrm{id}_{A} \otimes q: A \otimes B \rightarrow A \otimes Q$. Then clearly

$$
\left(T_{n} \otimes q\right)(x)=\left(T_{n} \otimes \mathrm{id}_{Q}\right)\left(\mathrm{id}_{A} \otimes q\right)(x)=0 .
$$

Given that

$$
\left(T_{n} \otimes \operatorname{id}_{Q}\right)\left(\operatorname{id}_{A} \otimes q\right)(x)=\left(\operatorname{id}_{A} \otimes q\right)\left(T_{n} \otimes \operatorname{id}_{B}\right)(x)
$$

we have that $\left(\operatorname{id}_{A} \otimes q\right)\left(T_{n} \otimes \mathrm{id}_{B}\right)(x)=0$. Since every operator $T_{n}$ is of finite rank, $\left(T_{n} \otimes \mathrm{id}_{B}\right)(x)$ belongs to the algebraic tensor product of $A \odot B$ of the algebras $A$ and $B$. The algebraic tensor product is an exact functor, so the vanishing condition

$$
\left(\operatorname{id}_{A} \otimes q\right)\left(T_{n} \otimes \operatorname{id}_{B}\right)(x)=0
$$

implies that $\left(T_{n} \otimes \operatorname{id}_{B}\right)(x)$ is an element of $A \otimes I$. Therefore

$$
x=\lim \left(T_{n} \otimes \operatorname{id}_{B}\right)(x)
$$

is also in $A \otimes I$.

This proves that the kernel of the map $\operatorname{id}_{A} \otimes q$ is identical to $A \otimes I$ for any algebras $B$ and $I$, which implies that $A$ is exact.

Thus if the reduced $C^{*}$-algebra of a discrete group has the CBAP then the group is exact and so it satisfies the Novikov conjecture. In particular, since the CPAP implies the CBAP, Lance's theorem implies that amenable groups satisfy the Novikov conjecture. On the other hand there exist exact groups that are not amenable, for example the free groups [13] and the word hyperbolic groups [26].

\section{The Metric Approximation Property}

In this section we will prove the following theorem.

Theorem 4.1. Let $\Gamma$ be a discrete group satisfying the rapid decay property with respect to a length function $\ell$ which is conditionally negative. Then the reduced $C^{*}$-algebra $C_{r}^{*}(\Gamma)$ has the metric approximation property.

The central point of our proof is an observation that the proof of the same property for free groups due to Haagerup [7] transfers directly to this more general situation. We also note that under the same hypotheses, the Fourier algebra $A(\Gamma)$ has a bounded approximate identity, which implies that it too has the MAP.

Following Haagerup [7, Def. 1.6] we say that a function $\phi: \Gamma \longrightarrow \mathbb{C}$ is a multiplier of $C_{r}^{*}(\Gamma)$ if and only if there exists a unique bounded 
operator $M_{\phi}: C_{r}^{*}(\Gamma) \rightarrow C_{r}^{*}(\Gamma)$ such that

$$
M_{\phi} \lambda(\gamma)=\phi(\gamma) \lambda(\gamma)
$$

for all $\gamma \in \Gamma$. This condition can be written equivalently as:

$$
M_{\phi} \lambda(f)=\lambda(\phi \cdot f) .
$$

An important situation in which such operators arise is given by the following lemma, which is a generalisation of [7, Lemma 1.7]; the proof is essentially identical to the original.

Lemma 4.2. Let $\Gamma$ be a discrete group equipped with a length function $\ell$. Assume that $(\Gamma, \ell)$ satisfies the rapid decay inequality for given $C, s>$ 0 .

Let $\phi$ be any function on $\Gamma$ such that

$$
K=\sup _{\gamma \in \Gamma}|\phi(\gamma)|(1+\ell(\gamma))^{s}<\infty .
$$

Then $\phi$ is a multiplier of $C_{r}^{*}(\Gamma)$ and $\left\|M_{\phi}\right\| \leq C K$.

In particular this holds for any element $f \in \mathbb{C} \Gamma$ and for any such element $M_{f}$ has finite rank.

Proof. We start by showing that property RD allows us to construct a family of multipliers for $C_{r}^{*}(\Gamma)$ with controlled operator norms. For any discrete group $\Gamma$ the characteristic function $\delta_{e}$ of the identity element $e$ of $\Gamma$ is the identity of the group ring $\mathbb{C} \Gamma$. Since $\delta_{e}$ is a unit vector in $\ell^{2}(\Gamma)$ we have that for any $f \in \mathbb{C} \Gamma,\|\lambda(f)\| \geq\left\|\lambda(f)\left(\delta_{e}\right)\right\|_{2}=\left\|f * \delta_{e}\right\|_{2}=$ $\|f\|_{2}$.

Then for any $f \in \mathbb{C} \Gamma$, the pointwise product $\phi \cdot f$ is also an element of $\mathbb{C} \Gamma$, so we can apply the rapid decay inequality to get:

$$
\begin{aligned}
\|\lambda(\phi \cdot f)\| & \leq C \sqrt{\sum_{\gamma \in \Gamma}|\phi(\gamma) f(\gamma)|^{2}(1+\ell(\gamma))^{2 s}} \\
& \leq C \sup _{\gamma \in \Gamma}\left\{|\phi(\gamma)|(1+\ell(\gamma))^{s}\right\} \sqrt{\sum_{\gamma \in \Gamma}|f(\gamma)|^{2}} \\
& =C K\|f\|_{2} .
\end{aligned}
$$

Putting together the two inequalities we have that

$$
\|\lambda(\phi \cdot f)\| \leq C K\|f\|_{2} \leq C K\|\lambda(f)\| .
$$

This shows that the map from $\mathbb{C} \Gamma$ to $C_{r}^{*}(\Gamma)$ which sends $\lambda(f)$ to $\lambda(\phi \cdot f)$ is continuous and so extends to a unique map $M_{\phi}: C_{r}^{*}(\Gamma) \rightarrow C_{r}^{*}(\Gamma)$ with the property that $M_{\phi} \lambda(f)=\lambda(\phi \cdot f)$.

It is also clear that $\left\|M_{\phi}\right\| \leq C K$. Finally it is clear that if $\phi$ has finite support then $M_{\phi}$ has finite rank.

We recall the definition of a conditionally negative kernel: 
Definition 4.3. A conditionally negative kernel on a set $V$ is a function $f: V \times V \longrightarrow \mathbb{R}$ such that for any finite subset $\left\{v_{1}, \ldots, v_{n}\right\} \subset V$ and any real numbers $\left\{\lambda_{1}, \ldots, \lambda_{n}\right\}$ such that $\sum_{i} \lambda_{i}=0$ the following inequality holds:

$$
\sum_{i, j} \lambda_{i} \lambda_{j} f\left(v_{i}, v_{j}\right) \leq 0
$$

A conditionally negative kernel on a group $G$ is a conditionally negative kernel on the set of elements of $G$ such that for any $g, h, k$ in $G$, $f(g h, g k)=f(h, k)$.

We now deduce

Theorem 4.4. Let $\Gamma$ be a discrete group with a conditionally negative length function $\ell$, which satisfies the property (RD) for $C, s>0$. Then there exists a net $\left\{\phi_{\alpha}\right\}$ of functions on $\Gamma$ with finite support such that

(1) For each $\alpha,\left\|M_{\phi_{\alpha}}\right\| \leq 1$;

(2) $\left\|M_{\phi_{\alpha}}(x)-x\right\| \rightarrow 0$ for all $x \in C_{r}^{*}(\Gamma)$.

Proof. Since the length function $\ell$ is conditionally negative, it follows from Schoenberg's lemma that for any $r>0$ the function $\phi_{r}(\gamma)=$ $e^{-r \ell(\gamma)}$ is of positive type. Thus by [7, Lemma 1.1] (see also [10, Lemma 3.2 and 3.5]), for every $r$ there exists a unique completely positive operator $M_{\phi_{r}}: C_{r}^{*}(\Gamma) \rightarrow C_{r}^{*}(\Gamma)$ such that $M_{\phi_{r}}(\lambda(\gamma))=\phi_{r}(\gamma) \lambda(\gamma)$ for all $\gamma \in \Gamma$ and $\left\|M_{\phi_{r}}\right\|=\phi_{r}(e)=1$.

Let us now define a family $\phi_{r, n}$ of finitely supported functions on $\Gamma$ by truncating the functions $\phi_{r}$ to balls of radius $n$ with respect to the length function $\ell$. For every $\gamma \in \Gamma$ we put:

$$
\phi_{r, n}(\gamma)= \begin{cases}e^{-r \ell(\gamma)}, & \text { if } \ell(\gamma) \leq n \\ 0, & \text { otherwise. }\end{cases}
$$

Since $e^{-x}(1+x)^{s} \rightarrow 0$ for any positive $s$ and $x \rightarrow \infty, \sup _{\gamma \in \Gamma}\left|\phi_{r}(\gamma)\right|(1+$ $\ell(\gamma))^{s}<\infty$. If we denote this finite number by $K$, then clearly $\sup _{\gamma \in \Gamma}\left|\phi_{r, n}(\gamma)\right|(1+\ell(\gamma))^{s} \leq K$. Thus, for every $r$ and $n$, these functions are multipliers of $C_{r}^{*}(\Gamma)$, and the corresponding operators $M_{\phi_{r}}$ and $M_{\phi_{r, n}}$ have norms bounded by $C K$. Since the functions $\phi_{r, n}$ have finite support, the corresponding operators $M_{\phi_{r, n}}$ are of finite rank.

On the other hand, since

$$
\left(\phi_{r}-\phi_{r, n}\right)(\gamma)= \begin{cases}0, & \ell(\gamma) \leq n \\ e^{-r \ell(\gamma)}, & \ell(\gamma)>n\end{cases}
$$

we have that

$$
\begin{aligned}
& \sup _{\gamma \in \Gamma}\left|\left(\phi_{r}-\phi_{r, n}\right)(\gamma)\right|(1+\ell(\gamma))^{s} \\
& =\sup _{\ell(\gamma)>n}\left|\left(\phi_{r}-\phi_{r, n}\right)(\gamma)\right|(1+\ell(\gamma))^{s} \\
& \leq K_{n}<\infty
\end{aligned}
$$


where $K_{n} \rightarrow 0$ as $n \rightarrow \infty$. Thus these functions are multipliers of $C_{r}^{*}(\Gamma)$ and the corresponding operators $M_{\phi_{r}-\phi_{r, n}}$ are such that $\left\|M_{\phi_{r}-\phi_{r, n}}\right\| \leq$ $C K_{n} \rightarrow 0$, as $n \rightarrow \infty$.

Since

$$
\left\|M_{\phi_{r}}-M_{\phi_{r, n}}\right\|=\left\|M_{\phi_{r}-\phi_{r, n}}\right\|
$$

we have $\left\|M_{\phi_{r}}-M_{\phi_{r, n}}\right\| \rightarrow 0$ as $n \rightarrow \infty$. This implies that $\left\|M_{\phi_{r, n}}\right\| \rightarrow$ $\left\|M_{\phi_{r}}\right\|=\phi_{r}(e)=1$.

To get the correct bound on the norm of these operators we introduce scaled functions:

$$
\rho_{r, n}=\frac{1}{\left\|M_{\phi_{r, n}}\right\|} \phi_{r, n}
$$

The algebraic identity satisfied by the multipliers, as stated in (2), guarantees that on $\lambda(\mathbb{C} \Gamma)$ we have the following identity

$$
M_{\rho_{r, n}}=\frac{1}{\left\|M_{\phi_{r, n}}\right\|} M_{\phi_{r, n}} .
$$

We now want to show that each operator $M_{\rho_{r, n}}$ is a finite rank contraction on $C_{r}^{*}(\Gamma)$ and that the strong operator closure of the family $\left\{M_{\rho_{r, n}}\right\}$ contains the identity map id $: C_{r}^{*}(\Gamma) \rightarrow C_{r}^{*}(\Gamma)$. This means that for every positive $\epsilon$ there exists an operator $M_{\rho_{r, n}}$ such that

$$
\left\|M_{\rho_{r, n}} x-x\right\|<\epsilon
$$

for all $x \in C_{r}^{*}(\Gamma)$.

First, a simple use of the triangle inequality leads to the following argument.

$$
\begin{aligned}
\left\|M_{\rho_{r, n}}-M_{\phi_{r}}\right\| & \leq\left\|M_{\rho_{r, n}}-M_{\phi_{r, n}}\right\|+\left\|M_{\phi_{r, n}}-M_{\phi_{r}}\right\| \\
& =\left\|\left(1-1 /\left\|M_{\phi_{r, n}}\right\|\right) M_{\phi_{r, n}}\right\|+\left\|M_{\phi_{r, n}}-M_{\phi_{r}}\right\| \\
& \rightarrow 0 \quad \text { as } n \rightarrow \infty
\end{aligned}
$$

Let $x \in C_{r}^{*}(\Gamma)$. Then $x$ is a limit of a sequence of elements $x_{m} \in$ $\lambda(\mathbb{C} \Gamma)$ so that $\left\|M_{\rho_{r, n}}(x)\right\|=\lim _{m \rightarrow \infty}\left\|M_{\rho_{r, n}}\left(x_{m}\right)\right\|$, and equation (3) implies that $\left\|M_{\rho_{r, n}}\left(x_{m}\right)\right\|=\left\|\frac{1}{\left\|M_{\phi_{r, n}}\right\|} M_{\phi_{r, n}}\left(x_{m}\right)\right\|$.

This leads to the following estimate:

$$
\begin{aligned}
\left\|M_{\rho_{r, n}}(x)\right\| & =\lim _{m \rightarrow \infty}\left\|\frac{1}{\left\|M_{\phi_{r, n}}\right\|} M_{\phi_{r, n}}\left(x_{m}\right)\right\| \\
& \leq \lim _{m \rightarrow \infty}\left\|\frac{1}{\left\|M_{\phi_{r, n}}\right\|} M_{\phi_{r, n}}\right\|\left\|x_{m}\right\|=\lim _{m \rightarrow \infty}\left\|x_{m}\right\|=\|x\| .
\end{aligned}
$$

It follows that $\left\|M_{\rho_{r, n}}\right\| \leq 1$.

Finally, it is clear that for any $\gamma \in \Gamma, e^{-r \ell(\gamma)} \rightarrow 1$ as $r \rightarrow 0$. Thus for any $x=\sum_{\gamma \in \Gamma} \mu_{\gamma} \lambda(\gamma) \in \mathbb{C} \Gamma$ we have

$$
M_{\phi_{r}}(x)=\sum \mu_{\gamma} \phi_{r}(\gamma) \lambda(\gamma)
$$


so that

$$
\begin{aligned}
\lim _{r \rightarrow 0} M_{\phi_{r}}(x) & =\lim _{r \rightarrow 0} \sum \mu_{\gamma} \phi_{r}(\gamma) \lambda(\gamma) \\
& =\sum \mu_{\gamma}\left(\lim _{r \rightarrow 0} \phi_{r}(\gamma)\right) \lambda(\gamma) \\
& =\sum \mu_{\gamma} \lambda(\gamma)=x
\end{aligned}
$$

Since any $x \in C_{r}^{*}(\Gamma)$ can be approximated by a sequence $x_{m} \in \lambda(\mathbb{C} \Gamma)$ we have

$$
\begin{aligned}
\left\|M_{\phi_{r}}(x)-x\right\| & \leq\left\|M_{\phi_{r}}(x)-M_{\phi_{r}}\left(x_{m}\right)\right\| \\
& +\left\|M_{\phi_{r}}\left(x_{m}\right)-x_{m}\right\|+\left\|x_{m}-x\right\|
\end{aligned}
$$

Given that $\left\|M_{\phi_{r}}\right\| \leq 1$ for all $r>0,\left\|M_{\phi_{r}}(x)-M_{\phi_{r}}\left(x_{m}\right)\right\| \leq \| x-$ $x_{m} \|<\epsilon / 3$ for all large enough $n$ and independently of $r$. Thus the sum of the first and third term of this sum can be made smaller than $(2 / 3) \epsilon$, for all $r>0$, and independently of $m$. Now equation (6) shows that, as $r \rightarrow 0, M_{\phi_{r}}\left(x_{m}\right)$ tends to $x_{m}$ so the middle term will be smaller than $\epsilon / 3$ for all sufficiently small $r$. Thus, for all sufficiently small $r>0$, $\left\|M_{\phi_{r}}(x)-x\right\|<\epsilon$ and so

$$
\left\|M_{\phi_{r}}(x)-x\right\| \rightarrow 0
$$

as $r \rightarrow 0$ for all $x \in C_{r}^{*}(\Gamma)$.

Let $\epsilon>0$. Then it follows from (4) that for every $r>0$ and all sufficiently large $n,\left\|M_{\rho_{r, n}}-M_{\phi_{r}}\right\|<\epsilon / 2$. Secondly, as we have just shown, for all sufficiently small $r,\left\|M_{\phi_{r}}(x)-x\right\|<\epsilon / 2$. Given that

$$
\left\|M_{\rho_{r, n}} x-x\right\| \leq\left\|M_{\rho_{r, n}} x-M_{\phi_{r}} x\right\|+\left\|M_{\phi_{r}}(x)-x\right\|
$$

for every $x \in C_{r}^{*}(\Gamma)$, the norm on the left hand side can be made smaller than $\epsilon$ by taking a sufficiently large $n$ and a sufficiently small $r>0$.

This means that the strong closure of the family $\mathfrak{M}=\left\{M_{\rho_{r, n}}\right\}$ of finite rank contractions contains the identity map on the algebra $C_{r}^{*}(\Gamma)$. This implies that there exists a net of finitely supported functions $\phi_{\alpha}$ with corresponding finite rank contractions $M_{\phi_{\alpha}} \in \mathfrak{M}$ such that $\left\|M_{\phi_{\alpha}} x-x\right\| \rightarrow 0$. This concludes the proof.

As a corollary we obtain the main result of this note.

Theorem 4.5. Let $\Gamma$ be a discrete group satisfying the rapid decay property with respect to a length function $\ell$ which is conditionally negative. Then the reduced $C^{*}$-algebra $C_{r}^{*}(\Gamma)$ has the metric approximation property.

Th class of CAT(0) cube complexes plays in important role in geometry and geometric group theory. A CAT(0) cube complex is a cell complex in which each cell is isometric to a unit Euclidean cube, the glueing maps are isometries and such that the natural path metric obtained by 
integrating path length piecewise satisfies the CAT(0) inequality described in [?]. Intuitively this last condition ensures that the geodesic triangles in the path metric space are no fatter than they would be in Euclidean space. This condition ensures (among many other things) that the space is uniquely geodesic and contractible. Now according to Niblo and Reeves [18] given a group acting on a CAT(0) cube complex we obtain a conditionally negative kernel on the group which gives rise to a conditionally negative length function. By results of Chatterji and Ruane [2] the group will have the rapid decay property with respect to this this length function provided that the action is properly discontinuous, stabilisers are uniformly bounded and the cube complex has finite dimension. Hence we obtain:

Corollary 4.6. Groups acting properly discontinuously on a finite dimensional $C A T(0)$ cube complex with uniformly bounded stabilisers have the metric approximation property.

This class of examples includes free groups, finitely generated Coxeter groups [19], and finitely generated right angled Artin groups for which the Salvetti complex is a CAT(0) cube complex. A rich class of interesting examples is furnished by Wise, [25], in which it is shown that many small cancellation groups act properly and co-compactly on CAT $(0)$ cube complexes. The examples include every finitely presented group satisfying the $\mathrm{B}(4)-\mathrm{T}(4)$ small cancellation condition and all those word-hyperbolic groups satisfying the $\mathrm{B}(6)$ condition.

Another class of examples where the main theorem applies is furnished by groups acting co-compactly and properly discontinuously on real or complex hyperbolic space. According to a result of Faraut and Harzallah [5] the natural metrics on these hyperbolic spaces are conditionally negative and they give rise to conditionally negative length functions on the groups. See [23] for a discussion and generalisation of this fact. The fact that these metrics satisfy rapid decay for the group was established by Jolissaint in [11].

Finally we remark that the net $\phi_{\alpha}$ of Theorem 4.4 provides an approximate identity for the Fourier algebra $A(\Gamma)$ of the group $\Gamma$ which is bounded in the multiplier norm. This implies, as in [7, Corollary 2.2], that if a group $\Gamma$ satisfies the $(\mathrm{RD})$ property with respect to a conditionally negative length function then its Fourier algebra $A(\Gamma)$ has the metric approximation property.

\section{REFERENCES}

1. J. Brodzki and R. J. Plymen, Periodic cyclic homology of certain nuclear algebras, C. R. Acad. Sci. Paris 329 (1999), 671-676.

2. I. Chatterji, K. Ruane, Some geometric groups with rapid decay, ArXiv preprint math.GR/0310356, http://xxx.lanl.gov/abs/math.GR/0310356.

3. J. de Canniere, U. Haagerup, Multipliers of the Fourier algebra of some simple Lie groups and their discrete subgroups, Amer. J. Math. 107 (1984), 455-500. 
4. P. Eymard, L'algèbre de Fourier d'un groupe localement compact. (French) Bull. Soc. Math. France 921964 181-236.

5. J. Faraut and K. Harzallah Distances hilbertiennes invariantes sur un espace homogène, Ann. Inst. Fourier (Grenoble) 24 (1974), no. 3, xiv, 171-217.

6. A. Grothendieck, Produits tensoriels topologiques et espaces nucléaires. Mem. Amer. Math. Soc. 1955 (1955), no. 16, 140 pp.

7. U. Haagerup, An example of a non-nuclear $C^{*}$-algebra which has the metric approximation property, Inventiones Math. 50 (1979), 279-293.

8. U. Haagerup, J. Kraus, Approximation properties for group $C^{*}$-algebras and group von Neumann algebras. Trans. Amer. Math. Soc. 344 (1994), no. 2, 667-699.

9. P. de la Harpe, Groupes hyperboliques, algébres d'operateurs et un theoréme de Jolissaint. C. R. Acad. Sci. Paris Ser. I 307 (1988), 771-774

10. P. de la Harpe, A. Valette, La propriété (T) de Kazhdan pour les groupes localement compacts, Asterisque 175 (1989), Soc. Mathématique de France.

11. P. Jolissaint. Rapidly decreasing functions in reduced $\mathrm{C}^{*}$-algebras of groups. Trans. Amer. Math. Soc. 317 (1990), 167-196.

12. P. Jolissaint, A. Valette, Normes de Sobolev et convoluteurs bornés sur $L^{2}(G)$, Ann. Inst. Fourier (Grenoble) 41 (1991), no. 4, 797-822.

13. E. Kirchberg, S. Wassermann, Exact groups and continuous bundles of $C^{*}-$ algebras. Math. Ann. 315 (1999), no. 2, 169-203.

14. V. Lafforgue, A proof of property (RD) for cocompact lattices of $\mathrm{SL}(3, \mathbf{R})$ and SL $(3$, C). J. Lie Theory 10 (2000), no. 2, 255-267

15. E. C. Lance, On nuclear $C^{*}$-algebras. J. Functional Analysis 12 (1973), 157176

16. H. Leptin, Sur l'algèbre de Fourier d'un groupe localement compact. (French) C. R. Acad. Sci. Paris Sr. A-B 2661968 A1180-A1182.

17. V. Mathai, Heat kernels and the range of the trace on completions of twisted group algebras. Contemporary Mathematics, (to appear) (2005).

18. G. A .Niblo, L. D. Reeves. The geometry of cube complexes and the complexity of their fundamental groups,Topology, Vol. 37, No 3, pp 621-633,1998.

19. G. A. Niblo and L. D. Reeves, Coxeter groups act on CAT(0) cube complexes, Journal of Group Theory, 6, (2003), pp 309-413.

20. V. Paulsen, Completely bounded maps and dilations. Pitman Research Notes in Mathematics Series, 146. Longman, New York, 1986.

21. R. J. Plymen, Reduced $C^{*}$-algebra of the p-adic group GL(n), J. Funct. Anal. 72 (1987), 1-12.

22. R. J. Plymen, Reduced $C^{*}$-algebra of the p-adic group $G L(n)$. II. J. Funct. Anal. 196 (2002), no. 1, 119-134.

23. G. Robertson, Crofton formulae and geodesic distance in hyperbolic spaces. J. Lie Theory 8 (1998), no. 1, 163-172.

24. S. Wassermann, Exact $C^{*}$-algebras and related topics, Lecture Notes Series, 19. Seoul National University, Research Institute of Mathematics, Global Analysis Research Center, Seoul, 1994.

25. Daniel T. Wise, Cubulating Small Cancellation Groups, Preprint, http://www.gidon.com/dani/tl.cgi?athe=pspapers/SmallCanCube.ps.

26. G. Yu, The coarse Baum-Connes conjecture for spaces which admit a uniform embedding into Hilbert space. Invent. Math. 139 (2000), no. 1, 201-240.

School of Mathematical Sciences, University of Southampton, SouthampTON SO17 1BJ

E-mail address: j.brodzki@soton.ac.uk, g.a.niblo@soton.ac.uk. 